\title{
Voriconazole efficacy against Candida glabrata and Candida krusei: preclinical data using a validated in vitro pharmacokineticl pharmacodynamic model
}

\author{
Maria-Ioanna Beredaki ${ }^{1}$, Panagiota-Christina Georgiou ${ }^{1}$, Maria Siopi ${ }^{1}$, Lamprini Kanioura ${ }^{2}$, \\ Maiken Cavling Arendrup ${ }^{3-5}$, Johan W. Mouton ${ }^{2}$ and Joseph Meletiadis ${ }^{1,2 *}$
}

${ }^{1}$ Clinical Microbiology Laboratory, Attikon University Hospital, Medical School, National and Kapodistrian University of Athens, Athens, Greece; ${ }^{2}$ Department of Medical Microbiology and Infectious Diseases, Erasmus Medical Center, Rotterdam, The Netherlands; ${ }^{3}$ Unit of Mycology, Statens Serum Institut, Copenhagen, Denmark; ${ }^{4}$ Department of Clinical Medicine, University of Copenhagen, Copenhagen,

Denmark; ${ }^{5}$ Department of Clinical Microbiology, University of Copenhagen, Copenhagen, Denmark

*Corresponding author. E-mail: jmeletiadis@med.uoa.gr

Received 11 July 2019; returned 29 August 2019; revised 5 September 2019; accepted 11 September 2019

\begin{abstract}
Background: Voriconazole exhibits in vitro activity against Candida glabrata and Candida krusei (EUCAST/CLSI epidemiological cut-off values $1 / 0.25$ and $1 / 0.5 \mathrm{mg} / \mathrm{L}$, respectively). Yet, EUCAST found insufficient evidence to set breakpoints for these species. We explored voriconazole pharmacodynamics (PD) in an in vitro dynamic model simulating human pharmacokinetics (PK).
\end{abstract}

\begin{abstract}
Methods: Four C. glabrata and three C. krusei isolates (voriconazole EUCAST and CLSI MICs of 0.03-2 mg/L) were tested in the PK/PD model simulating voriconazole exposures $\left(t_{1 / 2} \sim 6 \mathrm{~h} q 12 \mathrm{~h}\right.$ dosing for 3 days). PK/PD breakpoints were determined calculating the PTA for exposure indices $\mathrm{AAUC}_{0-24} / \mathrm{MIC}$ associated with half-maximal activity $\left(\mathrm{EI}_{50}\right)$ using Monte Carlo simulation analysis.
\end{abstract}

Results: Fungal load increased from $3.60 \pm 0.35$ to $8.41 \pm 0.24 \log _{10} \mathrm{cfu} / \mathrm{mL}$ in the drug-free control, with a maximum effect of $\sim 1 \log _{10}$ kill of $C$. glabrata and C. krusei isolates with MICs of 0.06 and $0.25 \mathrm{mg} / \mathrm{L}$, respectively, at high drug exposures. The $72 \mathrm{~h} \log _{10} \mathrm{cfu} / \mathrm{mL}$ change versus $f A \cup C_{0-24} / \mathrm{MIC}$ relationship followed a sigmoid curve for C. glabrata $\left(R^{2}=0.85-0.87\right)$ and C. krusei $\left(R^{2}=0.56-0.76\right)$ with $\mathrm{EI}_{50}$ of 49 (32-76) and 52 (33-78) fAUC/MIC for EUCAST and 55 (31-96) and 80 (42-152) fAUC/MIC for CLSI, respectively. The PTAs for C. glabrata and C. krusei isolates with EUCAST/CLSI MICs $\leq 0.125 / \leq 0.06 \mathrm{mg} / \mathrm{L}$ were $>95 \%$. Isolates with EUCAST/CLSI MICs of $0.25-1 /$ $0.125-0.5$ would require trough levels $1-4 \mathrm{mg} / \mathrm{L}$; isolates with higher MICs would not attain the corresponding PK/PD targets without reaching toxicity.

Conclusions: The in vitro PK/PD breakpoints for C. glabrata and C. krusei for EUCAST $(0.125 \mathrm{mg} / \mathrm{L})$ and CLSI $(0.06 \mathrm{mg} / \mathrm{L})$ bisected the WT populations. Trough levels of $>4 \mathrm{mg} / \mathrm{L}$, which are not clinically feasible, are necessary for efficacy against WT isolates.

\section{Introduction}

The incidence of candidaemia has increased in recent years due to numerous factors, most important being the exposure to broadspectrum antimicrobial agents, the use of aggressive therapies such as cancer chemotherapy, use of indwelling vascular catheters, neutropenia, mucosal colonization of Candida spp. and prior surgery. ${ }^{1,2}$ Although Candida albicans is the predominant cause of invasive candidiasis, Candida glabrata is the second most common species in northern Europe and America, and Candida krusei is an important pathogen in cancer patients and patients with prior fluconazole exposure. ${ }^{1,3}$
C. glabrata and C. krusei isolates demonstrate reduced susceptibility or resistance to fluconazole. Echinocandins and amphotericin B are thus first- and second-line agents, respectively, against these pathogens. ${ }^{3,4}$ These two classes of antifungals are available as IV formulations only and not suitable for outpatient therapy. Furthermore, echinocandin resistance rates up to 15\% among clinical isolates of $C$. glabrata have been reported in some settings, with a subset demonstrating an MDR phenotype, limiting the therapeutic options even further. ${ }^{5}$

Voriconazole exhibits potent in vitro antifungal activity against both C. glabrata and C. krusei isolates, with CLSI epidemiological cut-off values ( 0.25 and $0.5 \mathrm{mg} / \mathrm{L}$, respectively) being significantly 
lower than those of fluconazole ( 8 and $32 \mathrm{mg} / \mathrm{L}$, respectively) ${ }^{6}$ and close to the susceptibility breakpoint for voriconazole against C. albicans $(0.125 \mathrm{mg} / \mathrm{L}){ }^{7}$ However, EUCAST susceptibility breakpoints have not been established due to a paucity of efficacy data, ${ }^{8}$ and CLSI breakpoints have been proposed only for C. krusei $(0.5 \mathrm{mg} / \mathrm{L})$ although with limited clinical data. ${ }^{9}$ Due to the small number of patients with C. glabrata and C. krusei infections treated with voriconazole, clinical efficacy-pharmacokinetic (PK)-MIC data that might help describe exposure-effect relationships and determine clinical breakpoints are not foreseen to be collected in the future.

In vitro PK/pharmacodynamic (PD) models can offer an alternative reliable approach for the description of the exposure-effect relationships, the determination of PK/PD susceptibility breakpoints using Monte Carlo analysis and the estimation of target values for therapeutic drug monitoring (TDM). This is especially attractive in the case of voriconazole, which has complex PK properties and for which TDM is recognized as an important component to ensure effective, but not toxic, therapy. ${ }^{10}$ In the present study, a previously described in vitro PK/PD model ${ }^{11}$ was used in order to simulate human voriconazole PK against C. glabrata and C. krusei isolates with different voriconazole susceptibilities and to determine PK/PD susceptibility breakpoints for EUCAST and CLSI methodologies. Subsequently, voriconazole AUC and trough levels in serum necessary for optimal treatment and minimal toxicity were determined in relation to MICs.

\section{Materials and methods}

\section{Candida isolates}

Four clinical C. glabrata isolates and three clinical C. krusei isolates with increasing voriconazole EUCAST ${ }^{12}$ and $\mathrm{CLSI}^{13}$ MICs ranging from 0.03 to $2 \mathrm{mg} / \mathrm{L}$ were studied. The median EUCAST and CLSI MICs were determined after $24 \mathrm{~h}$ of incubation in triplicate experiments. The isolates were stored in normal sterile saline with $10 \%$ glycerol at $-70^{\circ} \mathrm{C}$ and revived by subculturing on Sabouraud dextrose agar plates supplemented with gentamicin and chloramphenicol (SDA; bioMérieux) to ensure purity and viability. Inoculum suspensions were prepared in normal sterile saline from $24 \mathrm{~h}$ cultures and adjusted to a final inoculum of $10^{4} \mathrm{cfu} / \mathrm{mL}$ using a counting chamber. The number of cfu was confirmed by quantitative cultures on SDA plates.

\section{Antifungal drugs and medium}

Pure powder of voriconazole (Pfizer Inc., Athens, Greece) was dissolved in sterile DMSO (Carlo Erba Reactifs-SDS, Val de Reuil, France) and stock solutions of $10 \mathrm{mg} / \mathrm{mL}$ were stored at $-70^{\circ} \mathrm{C}$ until use. The medium used in the in vitro PK/PD model was RPMI 1640 medium (with L-glutamine, without bicarbonate) buffered to $\mathrm{pH} 7.0$ with $0.165 \mathrm{M} \mathrm{MOPS}$ and supplemented with 100 mg/L chloramphenicol (AppliChem GmbH, Darmstadt, Germany).

\section{In vitro PK/PD model}

A previously described two-compartment PK/PD diffusion/dialysis model simulating in vivo $\mathrm{PK}^{11}$ was used. The model consists of an external compartment comprising a conical flask connected to a peristaltic pump (Minipuls Evolution ${ }^{\circledR}$, Gilson Inc.) and an internal compartment comprising a $10 \mathrm{~mL}$ volume semi-permeable cellulose dialysis tube (mol. wt $<20 \mathrm{kDa}$, Spectra/Por ${ }^{\circledR}$ Float-A-Lyzer ${ }^{\circledR}$ G2, Spectrum Laboratories Inc., Breda, The Netherlands) inoculated with a $10^{4} \mathrm{cfu} / \mathrm{mL}$ conidial suspension. Repeated sampling of $100 \mu \mathrm{L}$ was made from the internal compartment in order to ensure that drug concentrations in the internal compartment indeed mimic voriconazole drug concentration profiles in human plasma. Samples were stored at $-70^{\circ} \mathrm{C}$ until tested. Replicate experiments were conducted in order to assess the reproducibility.

\section{In vitro $P K$}

In order to describe the exposure-effect relationship, different voriconazole concentration-time profiles were simulated in the in vitro PK/PD model, with $f C_{\max }$ of $7,3.5,1.75$ and $0.8 \mathrm{mg} / \mathrm{L}$ and $t_{1 / 2}$ of $\sim 6 \mathrm{~h} .{ }^{14}$ Voriconazole levels were measured using a microbiological agar diffusion assay as previously described with a voriconazole-susceptible Candida parapsilosis isolate. ${ }^{15}$ The lowest limit of detection was $0.25 \mathrm{mg} / \mathrm{L}$ and intraday/interday variation was $<15 \%$. A concentration-time curve was generated for each simulated dose and analysed by non-linear regression analysis using a onecompartment model described by the equation $C_{t}=C_{0} e^{-k / t}$, where $C_{t}$ (dependent variable) is the concentration of drug at a given time ( $t$ ) (independent variable), $C_{0}$ is the initial concentration of the drug at $t=0 \mathrm{~h}, \mathrm{e}$ is the physical constant 2.18 and $k$ is the rate of drug removal. The $t_{1 / 2}$ was calculated using the equation $t_{1 / 2}=0.693 / \mathrm{k}$ and compared with the respective values observed in humans. Finally, the $\mathrm{AAUC}_{0-24}$ was calculated for each simulated dosage by applying the trapezoidal rule.

\section{In vitro PD}

To estimate the fungal load inside the dialysis tubes (internal compartment) of each voriconazole dosing regimen, $100 \mu \mathrm{L}$ samples were collected at regular intervals up to $72 \mathrm{~h}, 10$-fold serially diluted in normal saline and subcultured on SDA plates. Plates were incubated at $30^{\circ} \mathrm{C}$ for $24 \mathrm{~h}$ and colonies were counted at each dilution. Dilutions that yielded $10-50$ colonies were used in order to determine the $\log _{10} \mathrm{cfu} / \mathrm{mL}$ at each timepoint and to construct the time-kill curves. The lowest limit of detection was $10 \mathrm{cfu} / \mathrm{mL}$.

\section{PK/PD modelling}

To determine the in vitro exposure-response relationship, the $\log _{10} \mathrm{cfu} / \mathrm{mL}$ at $t=0 \mathrm{~h}$ was subtracted from the $\log _{10} \mathrm{cfu} / \mathrm{mL}$ at $72 \mathrm{~h}$ and plotted over the $f_{A U C} C_{-24} / \mathrm{MIC}$ ratio for each simulated dose and isolate. The data were then analysed with non-linear regression analysis using the sigmoidal model with variable slope $\left(E_{\max }\right.$ model) described by the equation $E=\left(E_{\max }-E_{\min }\right) \times E I^{n} /\left(E I^{n}+E I_{50}{ }^{n}\right)+E_{\min }$, where $E_{\max }$ is the maximum increase in $\log _{10} \mathrm{cfu} / \mathrm{mL}$ of the drug-free control (kept constant at $\log _{10} \mathrm{cfu} /$ $\mathrm{mL}$ in the drug-free control), $\mathrm{E}_{\min }$ is the minimum $\log _{10} \mathrm{cfu} / \mathrm{mL}$ found at high drug exposures (kept constant at $-1 \log _{10} \mathrm{cfu} / \mathrm{mL}$ ), EI is the exposure index $f A U C_{0-24} / \mathrm{MIC}, \mathrm{EI}_{50}$ is the exposure index required to achieve $50 \%$ of $E_{\max }-E_{\min }$ and $n$ is the slope of the dose-effect relationship (Hill coefficient). The goodness of fit of the $E_{\text {max }}$ model was assessed by visual inspection of graphs, residuals analysis, post run's test and $R^{2}$. All data were analysed using the statistics software package GraphPad Prism, version 5.0, for Windows (GraphPad Software, San Diego, CA, USA).

\section{Monte Carlo simulation}

Monte Carlo simulation analysis was performed using the Normal random number generator function of EXCEL (MS Office 2007) for 5000 patients receiving the standard IV voriconazole dosage of $4 \mathrm{mg} / \mathrm{kg}$ IV or $300 \mathrm{mg}$ orally twice daily, which corresponds to a total mean \pm SD steady-state $\mathrm{AUUC}_{0-12}$ of $50.4 \pm 41.83 \mathrm{mg} \cdot \mathrm{h} / \mathrm{L} .{ }^{14}$ For the simulation analysis, the $\mathrm{AAUC}_{0-24}$ used was calculated as $2 \times \mathrm{fAUC}_{0-12}$, where $\mathrm{fAUC}_{0-12}$ was $21.4 \pm 17.57 \mathrm{mg} \cdot \mathrm{h} / \mathrm{L}$ based on the $42 \%$ unbound fraction of voriconazole in human serum. ${ }^{16}$ The PTA for $\mathrm{EI}_{50}$ was estimated for isolates with MICs ranging from 0.008 to $4 \mathrm{mg} / \mathrm{L}$ and PK/PD susceptibility breakpoints were determined. Previously published MIC distribution data from $\mathrm{CLSI}^{17}$ and EUCAST ${ }^{8}$ were used. Since the uncertainty of $\mathrm{EI}_{50}$ is important for Monte Carlo simulations and PTA analysis, ${ }^{18}$ the PTAs were determined for the mean and the upper and lower 95\% CI limits of $\mathrm{EI}_{50}$ estimated with non-linear regression analysis. 


\section{Trough levels and MICs}

The required trough levels in human serum necessary to attain the $\mathrm{EI}_{50} \mathrm{~S}$ were calculated for different MICs. For that reason, the previously described relationship between serum tAUC and trough concentrations ( $\mathrm{t} C_{\mathrm{min}}$ ), namely tAUC $\mathrm{C}_{0-12}=7.011+12.687 \times \mathrm{t} C_{\mathrm{min}},{ }^{19}$ was used taking into account the $58 \%$ protein binding of voriconazole in human serum. ${ }^{16}$ The EUCAST and CLSI MICs for C. glabrata and C. krusei at which the corresponding PK/PD targets were attained were plotted against the $t A \cup C_{0-12}$ and $\mathrm{t}_{\mathrm{min}}$.

\section{Results}

\section{MICs}

The MICs for the included strains determined by EUCAST and CLSI methodologies are shown in Table 1. Most MICs fell within one 2-fold dilution when comparing across the EUCAST and CLSI methods, with an absolute agreement of $64 \%$.

\section{PK}

Figure 1 shows the steady-state plasma PK profiles based on human PK of twice-daily voriconazole dosages simulated in the

Table 1. Median (range) of triplicate MICs (mg/L) for EUCAST and CLSI for Candida isolates used in the present study

\begin{tabular}{lccc}
\hline Isolate no. & Reference code & EUCAST & CLSI \\
\hline C. glabrata 3 & SSI-W18236 & $0.06(0.03-0.125)$ & $0.03(0.03)$ \\
C. glabrata 5 & SSI-W17252 & $2(1-4)$ & $2(2)$ \\
C. glabrata 9 & SSI-W51696 & $0.5(0.125-2)$ & $0.5(0.5)$ \\
C. glabrata 11 & SSI-W42947 & $0.125(0.125-0.25)$ & $0.25(0.125-0.25)$ \\
C. krusei 2 & SSI-T5120 & $0.25(0.125-0.25)$ & $0.25(0.25)$ \\
C. krusei 4 & SSI-T3278 & $0.5(0.5)$ & $0.25(0.25)$ \\
C. krusei 12 & SSI-F49748 & $1(0.125-2)$ & $0.5(0.125-0.5)$ \\
\hline
\end{tabular}

SSI, Statens Serum Institut. in vitro PK/PD model. The mean $\pm \mathrm{SD} t_{1 / 2}$ was $6.9 \pm 2.7 \mathrm{~h}$, with $f C_{\max }$ of $6.49 \pm 0.98,3.84 \pm 0.30,1.76 \pm 0.60$ and $0.86 \pm 0.10 \mathrm{mg} / \mathrm{L}$, and AUC $_{0-24}$ of $47.71 \pm 9.97,28.12 \pm 5.82,12.13 \pm 3.80$ and $5.75 \pm$ $1.84 \mathrm{mg} \cdot \mathrm{h} / \mathrm{L}$, respectively, for all species and strains.

\section{PD}

C. glabrata (Figure 2) and C. krusei (Figure 3) isolates in the drugfree control grew from $3.60 \pm 0.35 \log _{10} \mathrm{cfu} / \mathrm{mL}$ at $0 \mathrm{~h}$ to $8.41 \pm 0.24$ $\log _{10} \mathrm{cfu} / \mathrm{mL}$ at $72 \mathrm{~h}$. Voriconazole decreased the fungal load in the tubes proportionally to the MIC for the isolates. The maximum effect of voriconazole corresponded to an $\sim 1 \log _{10} \mathrm{cfu} / \mathrm{mL}$ reduction from the initial inoculum. This was found at high voriconazole exposures for the isolates with the lowest EUCAST MIC, which was $0.06 \mathrm{mg} / \mathrm{L}$ for C. glabrata and $0.25 \mathrm{mg} / \mathrm{L}$ for C. krusei.

The $72 \mathrm{~h}$ change in the $\log _{10} \mathrm{cfu} / \mathrm{mL}$ versus $\mathrm{AAUC}_{0-24} / \mathrm{MIC}$ relationship for the C. glabrata and C. krusei isolates is displayed in Figure 4. The relationship followed a sigmoid curve for both species, but the relationship was much clearer for $C$. glabrata $\left(R^{2}=0.85-0.87\right)$ than for C. krusei $\left(R^{2}=0.56-0.76\right)$. For C. glabrata, curve fits and associated parameters of EUCAST- and CLSI-derived methods were comparable, with mean (95\% CI) $\mathrm{EI}_{50} \mathrm{~S}$ of 49 (32-76) and 55 (31-96) fAUC $_{0-24} / \mathrm{MIC}$, respectively. For C. krusei isolates, the difference in results according to susceptibility methods was more pronounced, with $\mathrm{EI}_{50}$ of 52 (33-78) for EUCAST and 80 (42-152) for CLSI. EI ${ }_{50}$ s determined from data at timepoints earlier than $72 \mathrm{~h}$ were different from those at $72 \mathrm{~h}$, in particular for the $24 \mathrm{~h}$ timepoint (data not shown). This was mainly due to the drug-free control not reaching its maximum growth (Figures 2 and 3).

\section{Monte carlo analysis}

The simulated patients had a mean \pm SD AAUC $_{0-24}$ of $41.94 \pm 35.41 \mathrm{mg} \cdot \mathrm{h} / \mathrm{L}$, very close to previously published voriconazole exposures. ${ }^{14}$ The PTAs for 49 (32-76) and 52 (33-78) fAUC $0-24$

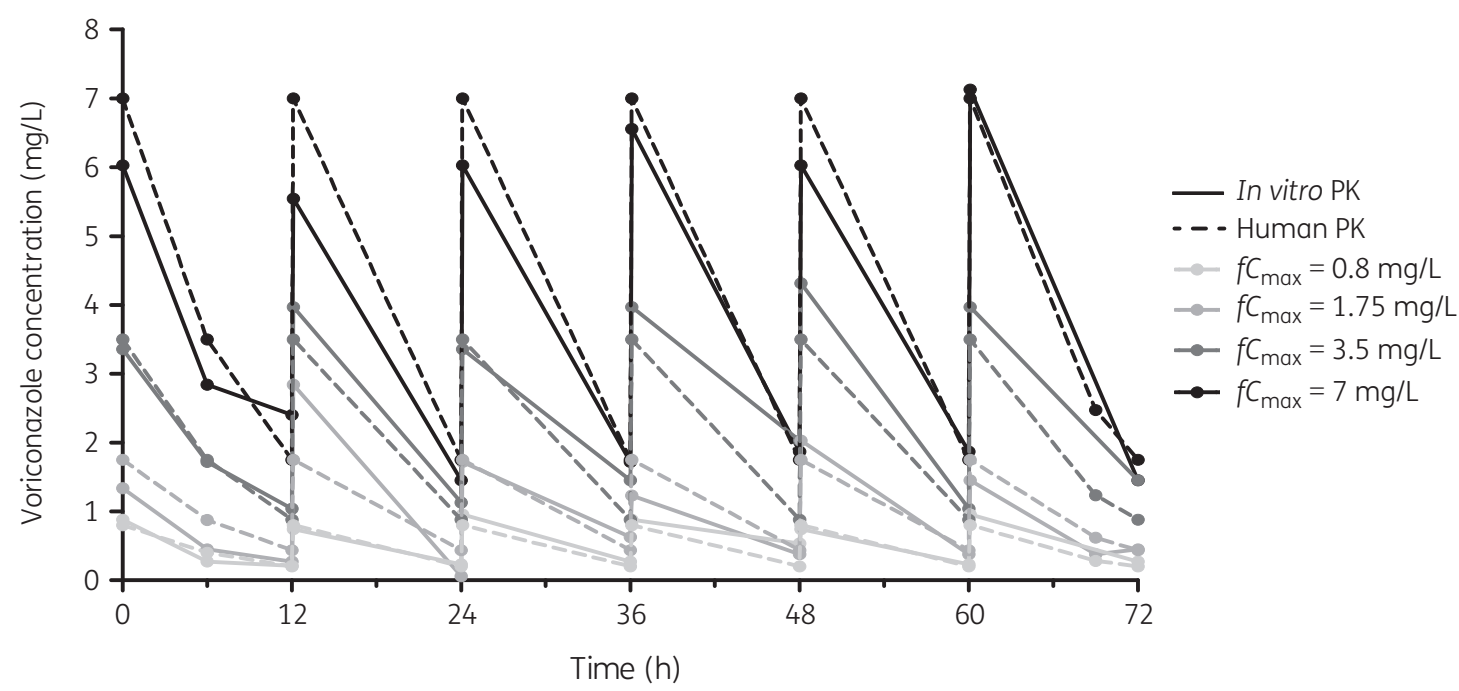

Figure 1. Representative concentration-time profiles of simulated $\mathrm{q} 12 \mathrm{~h}$ IV dosing regimens of voriconazole based on human PK in the in vitro PK/PD model with target $f C_{\max }$ of $0.8,1.75,3.5$ and $7 \mathrm{mg} / \mathrm{L}$ and obtained $C_{\min }$ of $0.23,0.67,1.04$ and $1.75 \mathrm{mg} / \mathrm{L}$, respectively, and $t_{1 / 2}$ of $\sim 6 \mathrm{~h}$. Data represent drug levels in the internal compartment of the in vitro model (solid lines) and the respective target values observed in human plasma (broken lines). 

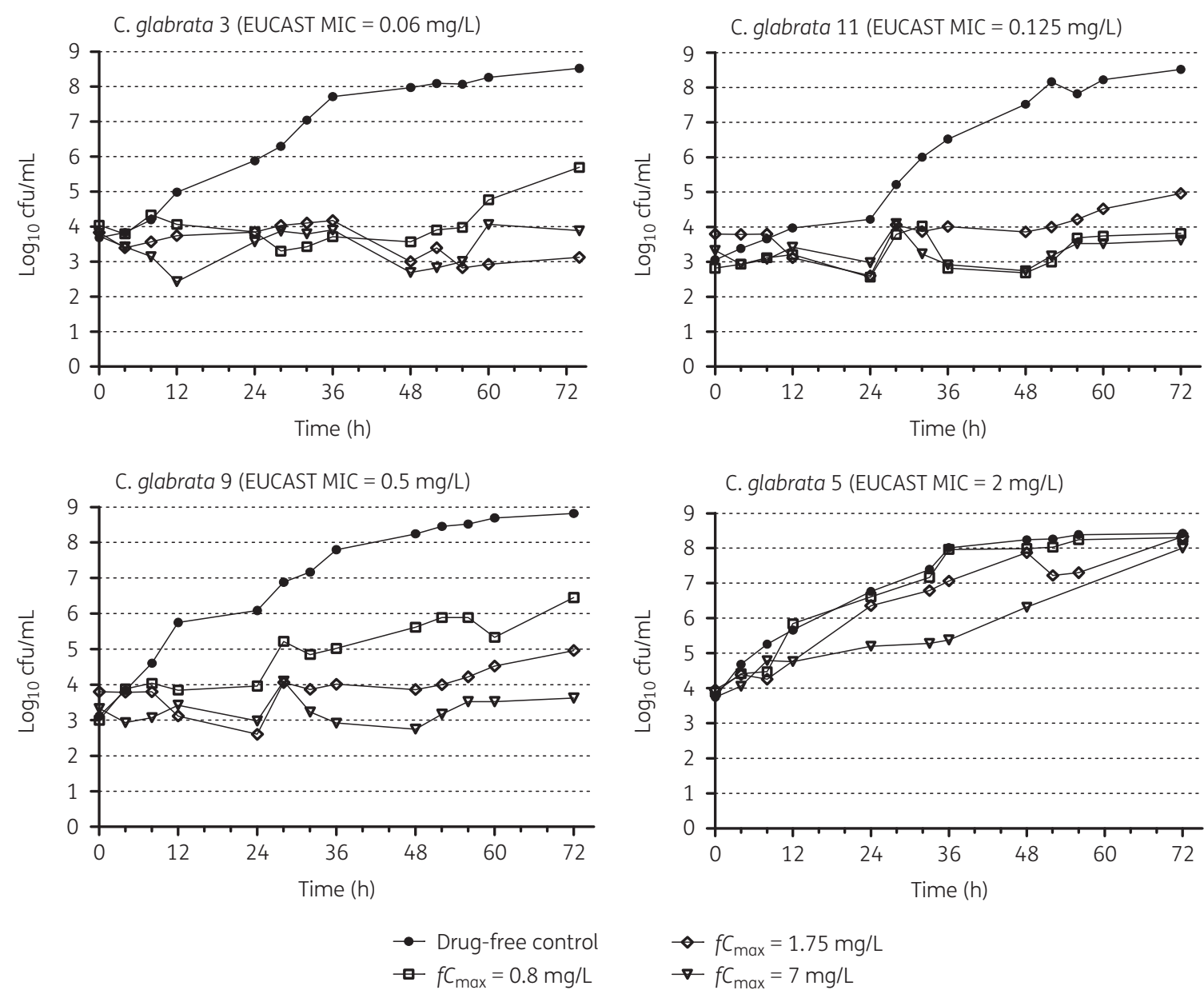

Figure 2. Time-kill curves in the in vitro PK/PD model simulating $q 12 \mathrm{~h}$ IV dosing regimens of voriconazole against $C$. glabrata isolates with $f C_{\text {max }}$ of $0.8,1.75$ and $7 \mathrm{mg} / \mathrm{L}$, and $t_{1 / 2}$ of $\sim 6 \mathrm{~h}$.

MIC for EUCAST and 55 (31-96) and 80 (42-152) fAUC $0-24 / \mathrm{MIC}$ for CLSI for C. glabrata and C. krusei isolates with increasing MICs are shown in Figure 5. The PTAs for the mean $\mathrm{EI}_{50}$ (solid black lines) were $>95 \%, 10 \%-95 \%$ and $<10 \%$ for C. glabrata and C. krusei isolates with EUCAST and CLSI MICs of $\leq 0.125,0.25-1$ and $\geq 2 \mathrm{mg} / \mathrm{L}$, whereas the PTAs for the upper $95 \%$ CI limit of $\mathrm{EI}_{50}$ (lower broken black lines) were $>95 \%, 10 \%-95 \%$ and $<10 \%$ for C. glabrata and C. krusei isolates with EUCAST MICs of $\leq 0.125,0.25-1$ and $\geq 2 \mathrm{mg} / \mathrm{L}$ and CLSI MICs of $\leq 0.06,0.125-0.5$ and $\geq 1 \mathrm{mg} / \mathrm{L}$, respectively (Figure 5).

\section{Trough levels and MICs}

The voriconazole trough levels in human serum required to attain the corresponding PK/PD targets for C. glabrata and C. krusei isolates with increasing EUCAST and CLSI MICs are shown in Figure 6. The corresponding PK/PD targets could be attained for C. glabrata and C. krusei isolates with EUCAST/CLSI MICs of 0.25-1/0.125$0.5 \mathrm{mg} / \mathrm{L}$, with trough levels of $1-4 \mathrm{mg} / \mathrm{L}$. In contrast, for isolates with higher MICs, trough levels of $>5.5 \mathrm{mg} / \mathrm{L}$, which are associated with increased risk of toxicity, ${ }^{10}$ will be required.

\section{Discussion}

The present study showed that voriconazole PK/PD targets cannot be attained for the vast majority of clinical C. glabrata and C. krusei isolates with the standard doses. Based on the in vitro PK/PD targets determined in the present study for C. glabrata and C. krusei, high PTAs were found only for isolates with EUCAST and CLSI MICS of $\leq 0.125$ and $\leq 0.06 \mathrm{mg} / \mathrm{L}$, respectively. These PK/PD breakpoints are two to three 2 -fold dilutions lower than the corresponding epidemiological cut-off values for EUCAST ( $1 \mathrm{mg} / \mathrm{L}$ for both species) and CLSI $(0.25 \mathrm{mg} / \mathrm{L}$ for C. glabrata and $0.5 \mathrm{mg} / \mathrm{L}$ for $\mathrm{C}$. krusei). This questions the efficacy of voriconazole for those infections.

Voriconazole produced a small killing effect against C. glabrata and C. krusei isolates ( $\sim 1 \log _{10}$ reduction) at high drug exposures against the most susceptible isolates also in line with previous in vitro PK/PD studies for low-MIC isolates. ${ }^{20-22}$ For C. glabrata there are no clinical breakpoints. The epidemiological cut-off values are $1 \mathrm{mg} / \mathrm{L}$ for $\mathrm{EUCAST}^{8}$ and $0.25 \mathrm{mg} / \mathrm{L}$ for CLSI. ${ }^{6}$ Based on the PK/PD target determined in the present study ( 50 fAUC $_{24} / \mathrm{MIC}$ ), the corresponding PK/PD susceptibility breakpoint of $\leq 0.125 \mathrm{mg} / \mathrm{L}$ for EUCAST and $\leq 0.06 \mathrm{mg} / \mathrm{L}$ for CLSI methodologies would bisect the WT MIC distributions. The PD target could be attained for isolates 
C. krusei 2 (EUCAST MIC $=0.25 \mathrm{mg} / \mathrm{L})$

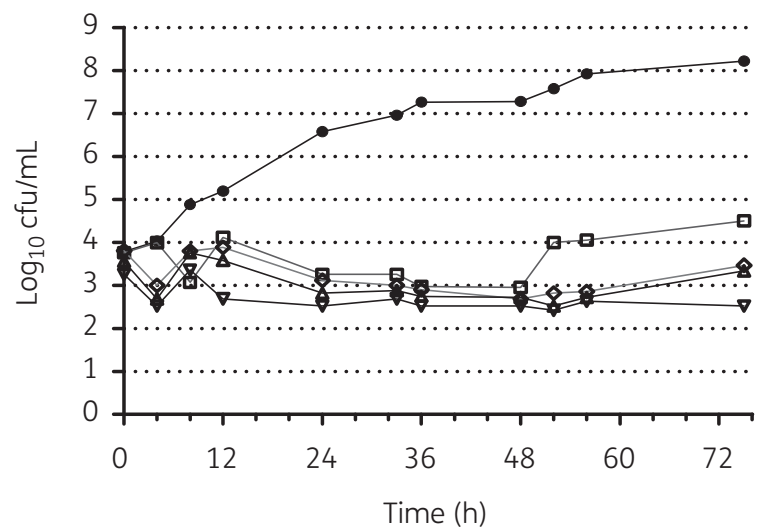

C. krusei 12 (EUCAST MIC $=1 \mathrm{mg} / \mathrm{L})$

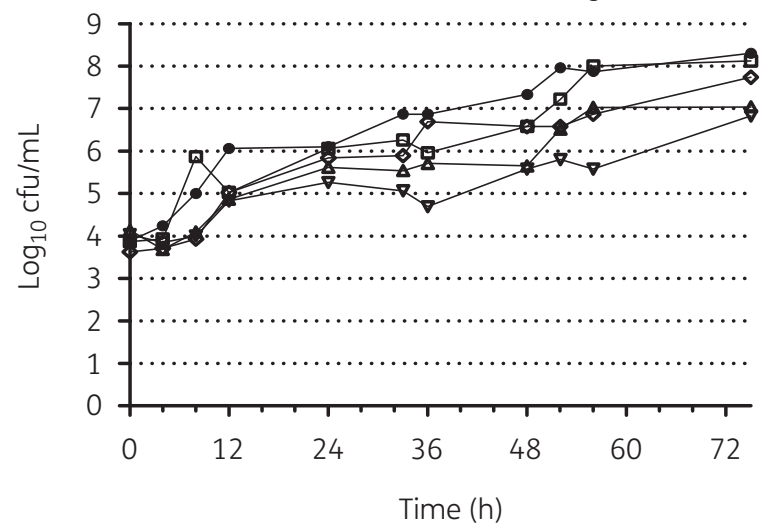

C. krusei 4 (EUCAST MIC $=0.5 \mathrm{mg} / \mathrm{L})$

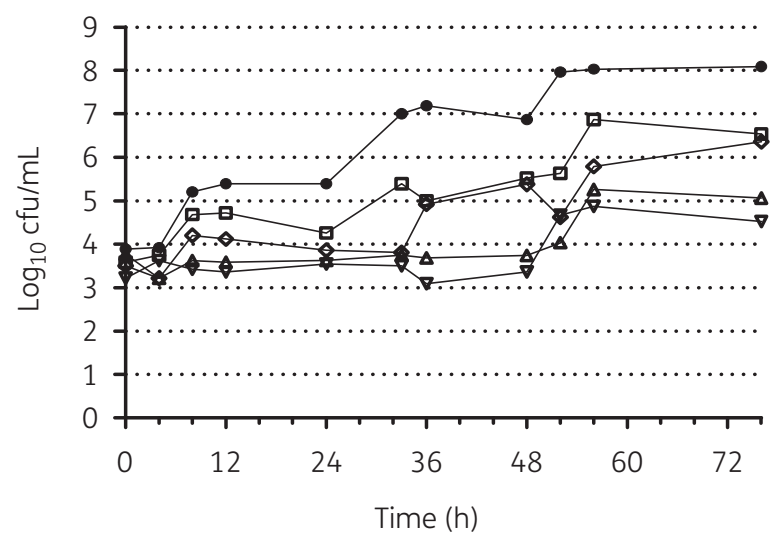

Figure 4. In vitro PK/PD relationship of voriconazole against $C$. glabrata and $C$. krusei isolates tested in the in vitro PK/PD model using EUCAST and CLSI MICs. The mean (95\% CI) fAUC ${ }_{0-24}$ /MICs are shown for each species for EUCAST and CLSI.

with EUCAST/CLSI MICs of $0.25-1 / 0.125-0.5 \mathrm{mg} / \mathrm{L}$, provided serum trough levels of at least $1-4 \mathrm{mg} / \mathrm{L}$ were ensured, thus necessitating TDM and dose adjustment in most cases. Previous animal studies showed that voriconazole at $40 \mathrm{mg} / \mathrm{kg}$ once daily was effective at reducing statistically significantly kidney fungal burden $>1 \log _{10}$ cfu/g (average $\sim 2 \log _{10} \mathrm{cfu} / \mathrm{g}$ ) compared with untreated controls after 7 days of treatment against C. glabrata isolates with CLSI MICs of $\leq 0.125 \mathrm{mg} / \mathrm{L}$ in grapefruit-fed neutropenic mice. ${ }^{23}$ 
(a)

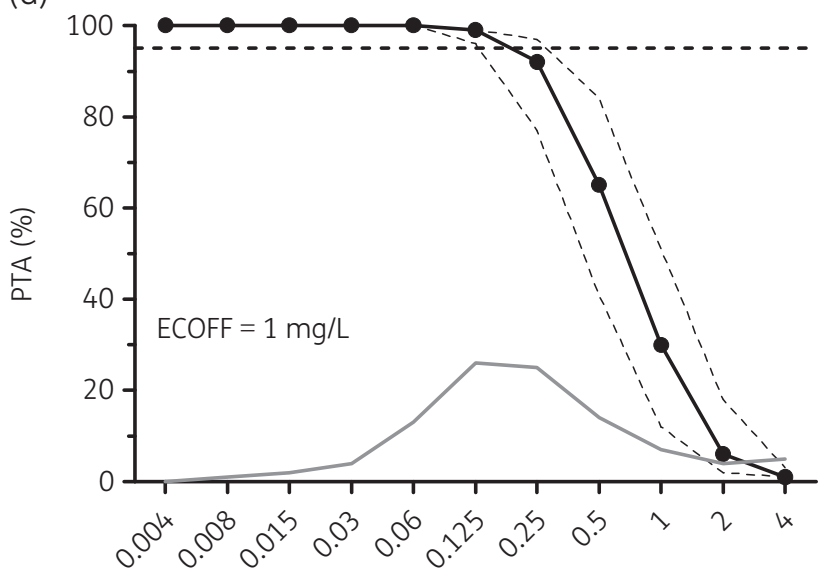

C. glabrata EUCAST MIC (mg/L)

(c)

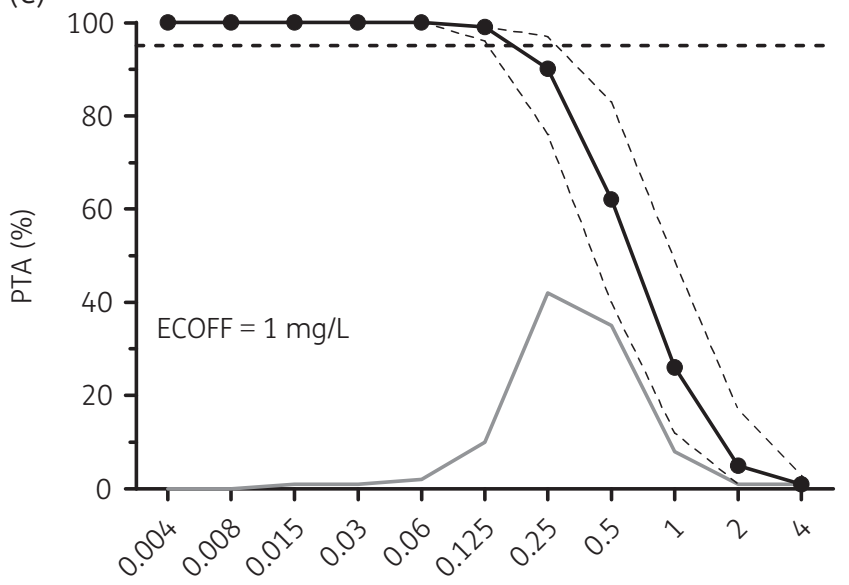

C. krusei EUCAST MIC (mg/L) (b)

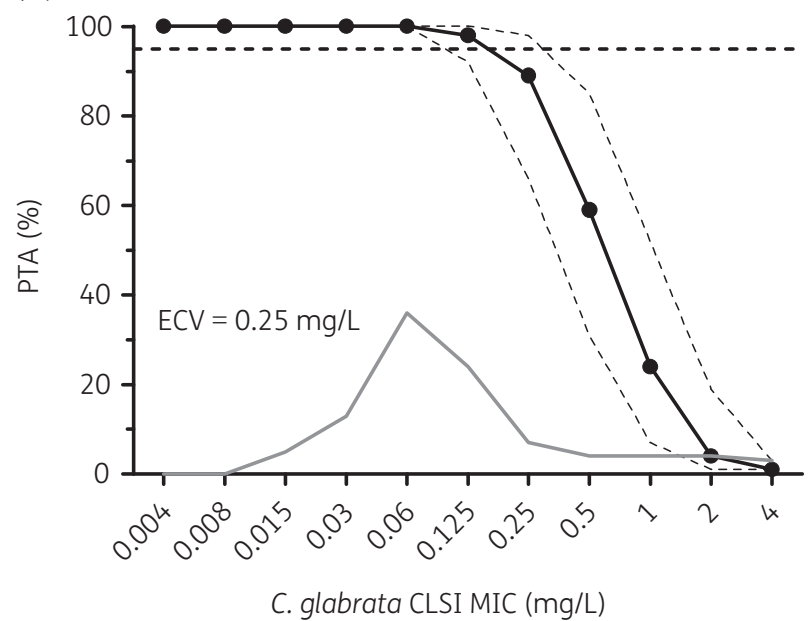

(d)

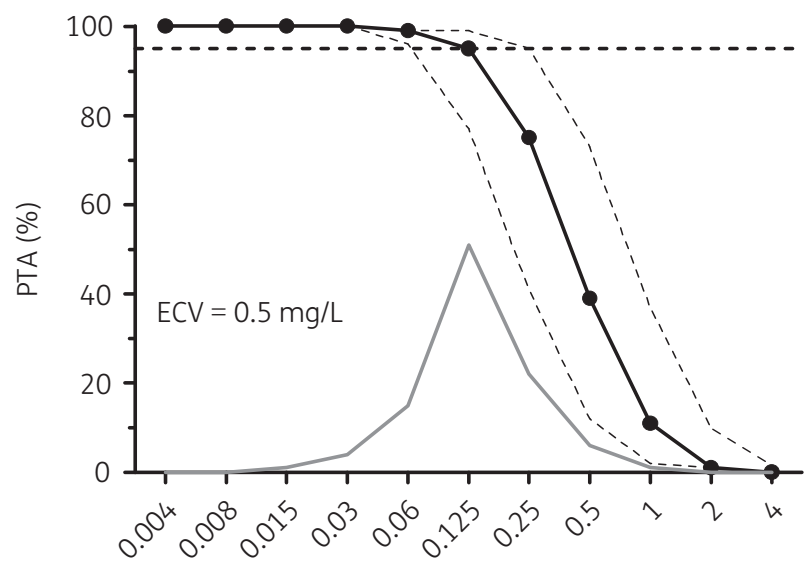

C. krusei CLSI MIC (mg/L)

Figure 5. PTA for 5000 patients receiving standard voriconazole dosages of $4 \mathrm{mg} / \mathrm{kg}$ IV twice daily for which the AUCs were simulated with Monte Carlo analysis for different EUCAST and CLSI MICs. Broken lines around the PTA curve represent the 95\% CI calculated using the 95\% CI limit of $\mathrm{EI}_{50}$ obtained from non-linear regression analysis of exposure-effect relationships for each Candida species. The horizontal broken line represents 95\% PTA. The epidemiological cut-off values for EUCAST (ECOFF) and CLSI (ECV) are shown for each species.

Voriconazole has been successfully used for C. glabrata infections, ${ }^{24,25}$ whereas breakthrough C. glabrata isolates have been reported in patients with voriconazole trough levels $\leq 0.63 \mathrm{mg} / \mathrm{L}^{26}$

For C. krusei, the PD targets of 52 fAUC $0-24 / M I C$ for EUCAST and 80 fAUC $_{0-24} / \mathrm{MIC}$ for CLSI were determined, suggesting a PK/PD breakpoint of 0.125 and $0.06 \mathrm{mg} / \mathrm{L}$, respectively, which are three 2 -fold dilutions lower that the corresponding epidemiological cut-off values of $1 \mathrm{mg} / \mathrm{L}$ (EUCAST) and $0.5 \mathrm{mg} / \mathrm{L}$ (CLSI). ${ }^{8,27}$ Currently, only CLSI has defined the susceptibility breakpoints of susceptible/intermediate/resistant (S/I/R) 0.5/1/2 mg/L although there are only a few cases $(n=9)$ of invasive candidiasis caused by C. krusei available for analysis. ${ }^{9}$ Of note, the response rate in the latter study was $78 \%$ for isolates with CLSI MICs of $0.125 \mathrm{mg} / \mathrm{L}$ (5/7) and $0.25 \mathrm{mg} / \mathrm{L}(2 / 2)$. However, the $0.125 \mathrm{mg} / \mathrm{L}$ breakpoint again bisects the WT distributions of EUCAST and CLSI methods. Voriconazole at 5 and $10 \mathrm{mg} / \mathrm{kg}$ twice daily was effective in reducing tissue fungal load by $1-2 \log _{10} \mathrm{cfu} / \mathrm{g}$ in neutropenic guinea pigs infected with C. krusei isolates with an MIC of $0.5 \mathrm{mg} / \mathrm{L}^{28}$
Considering that $10 \mathrm{mg} / \mathrm{kg}$ results in a tAUC $\tau$ of $31 \mathrm{mg} \cdot \mathrm{h} / \mathrm{L}$ and a protein binding of $45 \%,{ }^{29}$ the PK/PD target in the former study was around $68 \mathrm{FAUC}_{24} / \mathrm{MIC}$, which is close to the PK/PD target found in the present study. Voriconazole at $40 \mathrm{mg} / \mathrm{kg}$ once daily was effective in reducing tissue fungal load by 2 and $1 \log _{10} \mathrm{cfu} / \mathrm{g}$ compared with untreated controls of $C$. krusei isolates with CLSI MICs of 0.125 and $0.25 \mathrm{mg} / \mathrm{L}$, respectively, in grapefruit-fed neutropenic mice. ${ }^{30}$ Voriconazole has been successfully used for C. krusei infections, ${ }^{25,31}$ whereas breakthrough infection of $C$. krusei isolates has been reported in patients with trough levels of $0.53 \mathrm{mg} / \mathrm{L},{ }^{26}$ which hardly covers most isolates with MICs $>0.06 \mathrm{mg} / \mathrm{L}$ as shown in the present study.

Since in vitro data in artificial growth media simulating serum PK cannot fully compensate for the lack of clinical data, the clinical significance of the chosen $\mathrm{EI}_{50}$ endpoint, which corresponds to an $\sim 2 \log _{10} \mathrm{cfu} / \mathrm{mL}$ increase from the initial inoculum for azoles and Candida species, is unknown. Usually, stasis or 1 log kill is used, although again with no solid support for the clinical significance 
(a)

EUCAST and C.glabrata

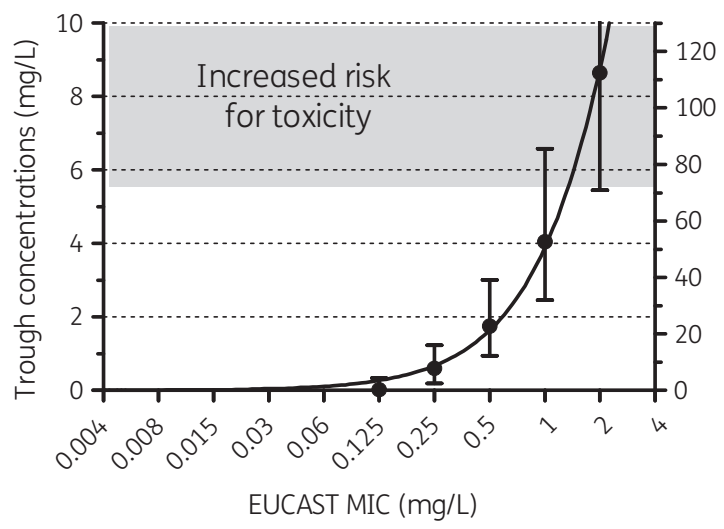

(c)

EUCAST and C.krusei

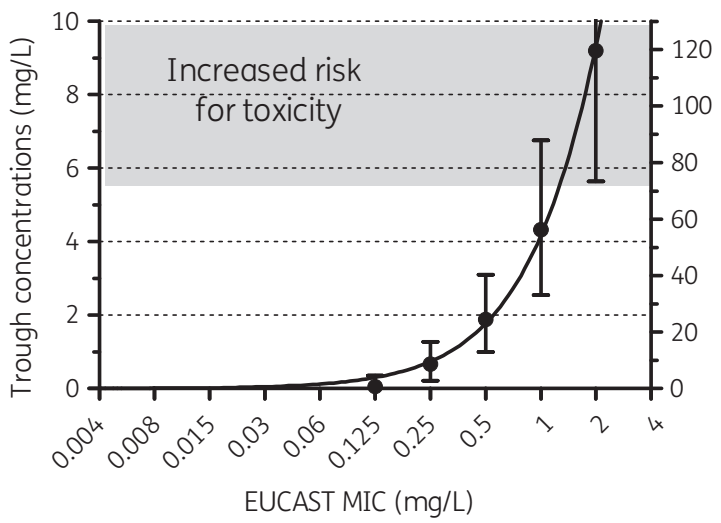

(b)

CLSI and C.glabrata

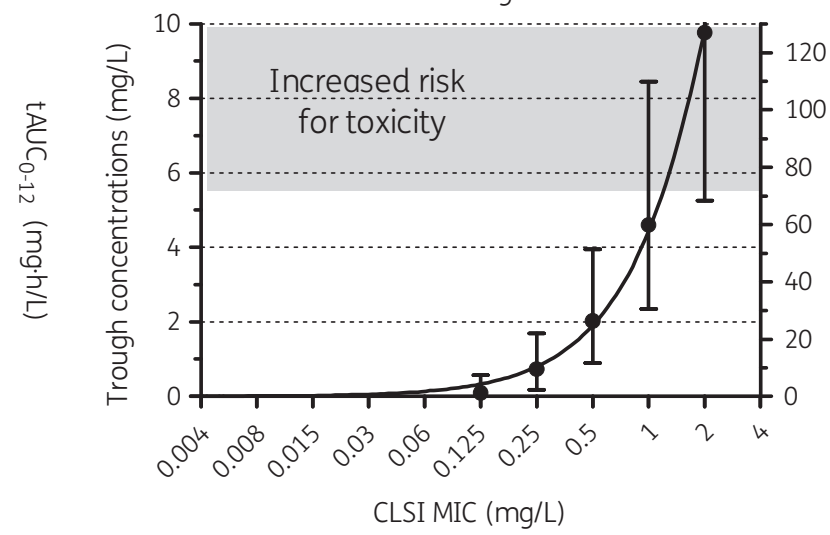

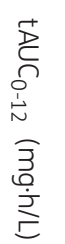

(d)

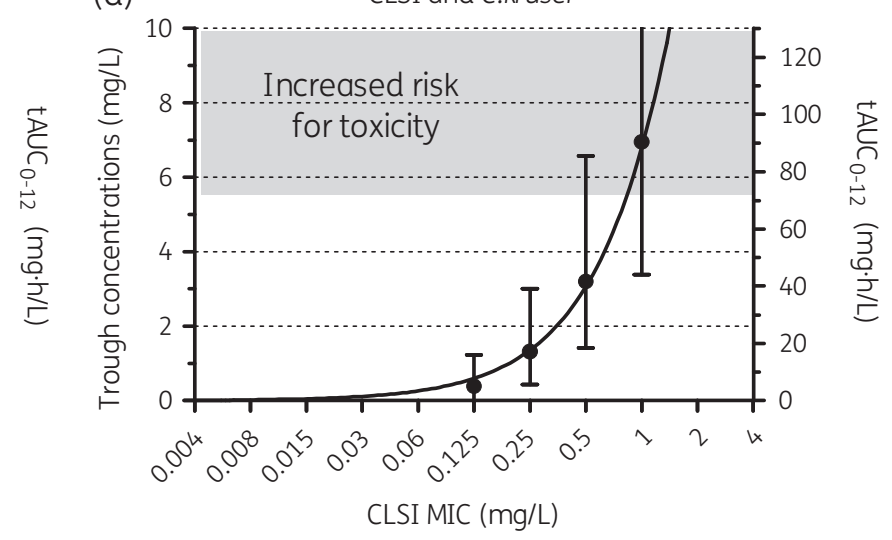

Figure 6. Correlation between voriconazole trough concentrations in human serum and EUCAST/CLSI MICs of C. glabrata and C. krusei in order to attain the corresponding PK/PD targets of 49 and 52 for EUCAST and 55 and 80 for CLSI, respectively. Error bars represent $95 \%$ CI.

of those effects. The $2 \log _{10} \mathrm{cfu} / \mathrm{mL}$ increase from the initial inoculum is further supported by the clinical AUC/MIC PD target of 100 for fluconazole, ${ }^{32,33}$ which corresponds to a $1-2 \log _{10}$ cfu/kidney increase from the initial inoculum in neutropenic animals. ${ }^{34}$ One explanation might be the absence of neutrophils both in in vitro and in neutropenic animal studies that usually exist in patients with invasive candidiasis, particularly in ICU patients. Indeed in vivo studies in neutropenic and non-neutropenic mice showed that median survival was prolonged and fungal load in kidney decreased by $1 \log _{10} \mathrm{cfu}$, whereas fluconazole reduced the fungal load by a further $1 \log _{10}$ cfu in non-neutropenic mice compared with neutropenic mice. ${ }^{35}$ In addition, voriconazole increased phagocytosis of Candida conidia by monocytes/polymorphonuclear leucocytes. ${ }^{36}$ Thus, the $2 \log _{10} \mathrm{cfu} / \mathrm{mL}$ increase from the initial inoculum in preclinical neutropenic models for azoles may result in clinical stasis.

The PK/PD breakpoints determined for $C$. glabrata and C. krusei for EUCAST $(0.125 \mathrm{mg} / \mathrm{L})$ and CLSI $(0.06 \mathrm{mg} / \mathrm{L})$ are symmetrical to the WT MIC distributions of both methodologies since they are two to three 2 -fold dilutions lower than EUCAST epidemiological cutoff values ( $1 \mathrm{mg} / \mathrm{L}$ for both species) and CLSI epidemiological cutoff values $(0.25 \mathrm{mg} / \mathrm{L}$ for $C$. glabrata and $0.5 \mathrm{mg} / \mathrm{L}$ for C. krusei). Of note, weighted analysis of $C$. glabrata MIC WT distribution indicated a CLSI epidemiological cut-off value of $0.5 \mathrm{mg} / \mathrm{L},{ }^{27}$ which increases symmetry of current PK/PD breakpoints for both species and methodologies. The PK/PD breakpoints cut exactly in the middle the WT C. glabrata MIC distribution and at the left side of the WT C. krusei MIC distribution for both EUCAST and CLSI methodologies. This implies that $47 \%$ of C. glabrata and $85 \%$ of C. krusei WT isolates with EUCAST MICs of $0.25-1 \mathrm{mg} / \mathrm{L}$ in previously published MIC distributions ${ }^{8}$ will be above the PK/PD breakpoint and, similarly, 31\% (35\% based on $0.5 \mathrm{mg} / \mathrm{L}$ CLSI epidemiological cutoff value) of C. glabrata and $79 \%$ of C. krusei WT isolates with CLSI MICs of $0.125-0.25$ and $0.125-0.5 \mathrm{mg} / \mathrm{L}$, respectively, in previously published CLSI MIC distributions. ${ }^{17}$ In addition, the modal MICs for EUCAST and CLSI MIC distributions differ by one 2-fold dilution (Figure 5), with the CLSI modal MIC being lower than the EUCAST modal MIC, ${ }^{37,38}$ further supporting the one dilution lower PK/PD susceptibility breakpoint of CLSI compared with EUCAST. However, one should also take into account the lower virulence of $C$. krusei compared with C. glabrata ${ }^{39}$ that may affect in vivo PD.

In conclusion, the present study suggests that voriconazole cannot be recommended for C. glabrata and C. krusei infections since the probability of attaining the PK/PD targets determined in the study is too low. The corresponding PK/PD targets for C. glabrata and C. krusei are 50-80 $\mathrm{AAUC}_{24} / \mathrm{MIC}$, resulting in a PK/PD susceptibility breakpoint of $\leq 0.125 \mathrm{mg} / \mathrm{L}$ for EUCAST and $\leq 0.06 \mathrm{mg} / \mathrm{L}$ for CLSI methodologies bisecting WT MIC distributions. 
Isolates with EUCAST and CLSI MICs $>1$ and $>0.5 \mathrm{mg} / \mathrm{L}$, respectively, should be considered resistant as toxic levels would be required to attain the PK/PD targets. For C. glabrata and C. krusei WT isolates with intermediate EUCAST/CLSI MICs of $0.25-1 / 0.125-0.5 \mathrm{mg} / \mathrm{L}$, TDM will be required to optimize drug exposure targeting trough levels of $1-4 \mathrm{mg} / \mathrm{L}$. However, due to the inherent variation in the susceptibility testing methods, in practice all WT isolates would require TDM to ensure that PK/PD targets are attained targeting trough concentrations of $>4 \mathrm{mg} / \mathrm{L}$ that will cover up to EUCAST and CLSI epidemiological cut-off values. This may be difficult in clinical practice because of a narrow therapeutic window $(4-5.5 \mathrm{mg} / \mathrm{L})$ and the large interindividual and interoccasion variability requiring real-time TDM and dose adjustment ${ }^{40}$ when no other alternatives exist (e.g. echinocandin-resistant infection in patients with kidney injury) or when a patient should be discharged with an oral therapy. However, those levels would be even more difficult to attain with the oral formulation $(300 \mathrm{mg} \mathrm{q12h}$ ) in haematological patients because of the $65 \%$ oral bioavailability. ${ }^{19}$ Since MIC clinical outcome data for C. glabrata and C. krusei species are difficult to collect, the findings of the present study provide a unique opportunity to propose PK/PD breakpoints and help define the role of voriconazole in the management of those infections.

\section{Funding}

This study was supported by an unrestricted grant from Pfizer, Greece and the ESCMID research grant 2016 (where ESCMID stands for European Society of Clinical Microbiology and Infectious Diseases).

\section{Transparency declarations}

None to declare.

\section{References}

1 Arendrup MC. Epidemiology of invasive candidiasis. Curr Opin Crit Care 2010; 16: 445-52.

2 Eggimann P, Garbino J, Pittet D. Epidemiology of Candida species infections in critically ill non-immunosuppressed patients. Lancet Infect Dis 2003; 3: 685-702.

3 Pappas PG, Kauffman CA, Andes DR et al. Clinical practice guideline for the management of candidiasis: 2016 update by the Infectious Diseases Society of America. Clin Infect Dis 2015; 62: e1-50.

4 Cornely OA, Bassetti M, Calandra Tet al. ESCMID guideline for the diagnosis and management of Candida diseases 2012: non-neutropenic adult patients. Clin Microbiol Infect 2012; 18 Suppl 7: 19-37.

5 Alexander BD, Johnson MD, Pfeiffer CD et al. Increasing echinocandin resistance in Candida glabrata: clinical failure correlates with presence of FKS mutations and elevated minimum inhibitory concentrations. Clin Infect Dis 2013; 56: 1724-32.

6 CLSI. Epidemiological Cutoff Values for Antifungal Susceptibility TestingSecond Edition: M59. 2018.

7 CLSI. Performance Standards for Antifungal Susceptibility Testing of Yeasts_First Edition: M60. 2017.

8 EUCAST. Voriconazole: Rationale for the EUCAST Clinical Breakpoints, Version 3.0. 2017.

9 Pfaller MA, Andes D, Arendrup MC et al. Clinical breakpoints for voriconazole and Candida spp. revisited: review of microbiologic, molecular, pharmacodynamic, and clinical data as they pertain to the development of speciesspecific interpretive criteria. Diagn Microbiol Infect Dis 2011; 70: 330-43.
10 Pascual A, Calandra T, Bolay S et al. Voriconazole therapeutic drug monitoring in patients with invasive mycoses improves efficacy and safety outcomes. Clin Infect Dis 2008; 46: 201-11.

11 Meletiadis J, Al-Saigh R, Velegraki A et al. Pharmacodynamic effects of simulated standard doses of antifungal drugs against Aspergillus species in a new in vitro pharmacokinetic/pharmacodynamic model. Antimicrob Agents Chemother 2012; 56: 403-10.

12 Arendrup MC, Guinea J, Cuenca-Estrella $M$ et al. EUCAST DEFINITIVE DOCUMENT E.DEF 7.3: Method for the Determination of Broth Dilution Minimum Inhibitory Concentrations of Antifungal Agents for Yeasts. 2015. http://www.eucast.org/fileadmin/src/media/PDFs/EUCAST_files/AFST/ Files/EUCAST_E_Def_7_3_Yeast_testing_definitive.pdf.

13 CLSI. Reference Method for Broth Dilution Antifungal Susceptibility Testing of Yeasts-Third Edition: M27. 2008.

14 Pfizer Inc. VFEND ${ }^{\circledR}$ (Voriconazole) United States Package Insert. New York, NY, USA: Pfizer Inc., 2010.

15 Siopi M, Neroutsos E, Zisaki K et al. Bioassay for determining voriconazole serum levels in patients receiving combination therapy with echinocandins. Antimicrob Agents Chemother 2016; 60: 632-6.

16 Purkins L, Wood N, Greenhalgh K et al. The pharmacokinetics and safety of intravenous voriconazole-a novel wide-spectrum antifungal agent. $\mathrm{Br} J$ Clin Pharmacol 2003; 56 Suppl 1: 2-9.

17 Espinel-Ingroff A, Pfaller MA, Bustamante B et al. Multilaboratory study of epidemiological cutoff values for detection of resistance in eight Candida species to fluconazole, posaconazole, and voriconazole. Antimicrob Agents Chemother 2014; 58: 2006-12.

18 Colin P, Eleveld DJ, Jonckheere S et al. What about confidence intervals? A word of caution when interpreting PTA simulations. J Antimicrob Chemother 2016; 71: 2502-8.

19 Liu P, Mould DR. Population pharmacokinetic analysis of voriconazole and anidulafungin in adult patients with invasive aspergillosis. Antimicrob Agents Chemother 2014; 58: 4718-26.

$20 \mathrm{Li}$ Y, Nguyen MH, Derendorf H et al. Measurement of voriconazole activity against Candida albicans, C. glabrata, and C. parapsilosis isolates using timekill methods validated by high-performance liquid chromatography. Antimicrob Agents Chemother 2007; 51: 2985-7.

21 Li Y, Nguyen MH, Cheng S et al. A pharmacokinetic/pharmacodynamic mathematical model accurately describes the activity of voriconazole against Candida spp. in vitro. Int J Antimicrob Agents 2008; 31: 369-74.

22 Klepser ME, Malone D, Lewis RE et al. Evaluation of voriconazole pharmacodynamics using time-kill methodology. Antimicrob Agents Chemother 2000; 44: 1917-20.

23 Sanchis M, Capilla J, Castanheira M et al. Voriconazole minimum inhibitory concentrations are predictive of treatment outcome in experimental murine infections by Candida glabrata. Int J Antimicrob Agents 2016; 47: 286-8.

24 Einarsdóttir HM, Danielsen R, Gottfredsson M. Successful treatment of Candida glabrata myocarditis with voriconazole. Scand J Infect Dis 2002; 34: 778-80.

25 Ostrosky-Zeichner L, Oude Lashof AML, Kullberg BJ et al. Voriconazole salvage treatment of invasive candidiasis. Eur J Clin Microbiol Infect Dis 2003; 22: 651-5.

26 Trifilio S, Singhal S, Williams S et al. Breakthrough fungal infections after allogeneic hematopoietic stem cell transplantation in patients on prophylactic voriconazole. Bone Marrow Transpl 2007; 40: 451-6.

27 Espinel-Ingroff A, Turnidge J. The role of epidemiological cutoff values (ECVs/ECOFFs) in antifungal susceptibility testing and interpretation for uncommon yeasts and moulds. Rev Iberoam Micol 2016; 33: 63-75.

28 Ghannoum M, Okogbule-Wonodi I, Bhat $N$ et al. Antifungal activity of voriconazole (UK-109, 496), fluconazole and amphotericin B against hematogenous Candida krusei infection in neutropenic guinea pig model. J Chemother 1999; 11: 34-9. 
29 Roffey SJ, Cole S, Comby P et al. The disposition of voriconazole in mouse, rat, rabbit, guinea pig, dog, and human. Drug Metab Dispos 2003; 31: 731-41.

30 Marine M, Pastor FJ, Serena C et al. Efficacy of triazoles in a murine disseminated infection by Candida krusei. Antimicrob Agents Chemother 2009; 53: 3585-8.

31 Sili U, Yilmaz M, Ferhanoglu B et al. Candida krusei arthritis in a patient with hematologic malignancy: successful treatment with voriconazole. Clin Infect Dis 2007; 45: 897-8.

32 EUCAST. Fluconazole: Rationale for the EUCAST Clinical Breakpoints, Version 2.0. 2013. http://www.eucast.org/fileadmin/src/media/PDFs/EUCAST_ files/Rationale_documents/Fluconazole_rationale_2_0_20130223.pdf.

33 Pfaller MA, Andes D, Diekema DJ et al. Wild-type MIC distributions, epidemiological cutoff values and species-specific clinical breakpoints for fluconazole and Candida: time for harmonization of CLSI and EUCAST broth microdilution methods. Drug Resist Updat 2010; 13: 180-95.

34 Andes D, van Ogtrop M. Characterization and quantitation of the pharmacodynamics of fluconazole in a neutropenic murine disseminated candidiasis infection model. Antimicrob Agents Chemother 1999; 43: 2116-20.
35 Wiederhold NP, Najvar LK, Bocanegra R et al. Comparison of anidulafungin's and fluconazole's in vivo activity in neutropenic and non-neutropenic models of invasive candidiasis. Clin Microbiol Infect 2012; 18: E20-3.

36 Katragkou A, Kruhlak MJ, Simitsopoulou M et al. Interactions between human phagocytes and Candida albicans biofilms alone and in combination with antifungal agents. J Infect Dis 2010; 201: 1941-9.

37 Pfaller MA, Castanheira M, Messer SA et al. Comparison of EUCAST and CLSI broth microdilution methods for the susceptibility testing of 10 systemically active antifungal agents when tested against Candida spp. Diagn Microbiol Infect Dis 2014; 79: 198-204.

38 Pfaller MA, Espinel-Ingroff A, Boyken $L$ et al. Comparison of the broth microdilution (BMD) method of the European Committee on Antimicrobial Susceptibility Testing with the 24-hour CLSI BMD method for testing susceptibility of Candida species to fluconazole, posaconazole, and voriconazole by use of epidemiological cutoff values. J Clin Microbiol 2011; 49: 845-50.

39 Arendrup M, Horn T, Frimodt-Møller N. In vivo pathogenicity of eight medically relevant Candida species in an animal model. Infection 2002; 30: 286-91.

40 Rawson TM, Herrero P, Sharma S et al. Delivering precision antimicrobial therapy through closed-loop control systems. J Antimicrob Chemother 2018; 73: 835-43. 